\title{
Normalization and Matching in the DORO System
}

\author{
C.H.A. Koster, C. Derksen, D. van de Ende and J. Potjer \\ Department of Computer Science, \\ University of Nijmegen, The Netherlands. \\ $\{$ kees, caspard, dvdende, jasperp\}@cs.kun.nl
}

\begin{abstract}
This paper is concerned with the use of linguistically motivated phrases as indexing terms in Information Retrieval applications. Apart from the conventional noun phrases, we propose to use verb phrases as index terms for text classification. Techniques for phrase matching through syntactic normalization and semantical matching are described. We discuss the realization of the syntactic normalization of phrases by transduction to frames. Semantical normalization is based on lexico-semantical relations, taking into account certain properties of the classification algorithms used.

The ideas described here are being implemented in the Document Routing system DORO, in which statistical learning algorithms are applied to document profiles consisting of phrases. This paper describes the rationale behind work in progress, rather than presenting final results.
\end{abstract}

\section{Introduction}

In the Information Retrieval community there is a long standing debate concerning the value of linguistic techniques. Although the use of simple noun phrases as indexing terms is now commonly accepted, practical Information Retrieval systems using phrases like the CLARIT system [5] do not appear to perform consistently better than those based on keywords. There is a widespread conviction that the value of NLP to IR is dubious, even among people who tried hard to make it work [14].

In this paper we describe the techniques developed in the DORO project for the extraction, normalization and matching of phrases. One of the aims of the DORO project is to show that the use of phrases is advantageous in the routing situation, where documents are to be classified on the basis of a superficial analysis of their contents.

The routing (or filtering) situation presents a good setting for the experimentation with linguistic techniques in IR, since the vexing problem of computing Recall is much easier to solve than in the classical retrieval situation. Preclassified training and testing documents are readily available. Classification experiments can be performed completely automatically, without involving human judgement. The number of documents needed for a meaningful experiment is much smaller than the number of documents in a realistic database, the number of classes much smaller than the number of possible queries in an IR system, and the classes are known in advance. Therefore the routing problem makes it simple to experiment with the effect of other document representations than the traditional keywords.

\subsection{Shallow and deep linguistics}

A distinction is often made between "shallow" and "deep" linguistic techniques employed in IR. By a shallow technique is meant one which needs few linguistic resources and little linguistic knowledge in order to be used. Many shallow techniques are routinely used in IR:

- stemming [9] in order to eliminate morphological variation and thus enhance Recall

- considering function words (closed syntactical categories) as stop words

- expansion of a query with conceptually related words. 
The most advanced shallow techniques used are noun phrase pickers and Part-Of-Speech taggers. Typically, the resources for shallow techniques can be obtained by a cry for help on a suitable mailing list.

On the other hand, linguistics offers many more concepts and ideas which could be applied, but which are harder to put into a tool and to use without extensive linguistic knowledge. Deriving, representing and searching the content of documents is still very far away. Even a reliable parser for English with a large lexicon, high coverage and acceptable speed is not available in the public domain. It is hard to develop such a parser (outside of limited domains), not easy to make it work in an IR context and even harder to maintain and extend it.

The very success of the shallower linguistic techniques in conjunction with statistical techniques (see e.g. [15]) is a barrier for the use of deep techniques in IR: it is hard to justify the development costs.

In the DORO system, a complete syntactical analysis is made of the documents, in order to extract phrases. This requires the development of grammars and lexica for a number of languages and a parser generator system generating efficient and robust parsers. Given the present state-of-the-art in IR, this is deep linguistics.

\subsection{The KeyPhrase hypothesis}

Most of the commercial Information Retrieval systems are based, explicitly or implicitly, on what we shall call the KeyWord hypothesis:

A document is a bag of words.

A query is a bag of words.

The more words the query and the document have in common, the more the document is about the query.

Our work in the application of NLP techniques to the classification of documents is based on the KeyPhrase hypothesis (see [1]):

A document is a bag of phrases.

A query is a bag of phrases.

The more phrases the query and the document have in common, the more the document is about the query.

The predictive value of single keywords has its limits, not only in classical Information Retrieval but also in Text Classification. Experiments by others (e.g. [2]) have shown that by using combinations of keywords rather than single keywords a better classification may be achieved. We are interested in Text Classification using linguistically meaningful phrases as terms: not only the Noun Phrase (NP) including its modifiers but also the Verb Phrase (VP) including its complements.

The Noun Phrase describes a complicated concept, in the form of a head with modifiers. Whenever the head alone is not precise enough, it is modified by one or more adjectives, nouns or preposition phrases. Similarly the Verb Phrase describes a situation or process by relating a main verb to a number of NP's and other phrases (like a small semantical network).

Our initial experiments (Arampatzis, to be published) have shown that:

1. using only NP heads and main verbs as terms, the error rate of classification is somewhat worse than when using all keywords. The modifiers are essential to achieve precision.

2. using complete NP's and VP's as terms, the error rate of classification is also worse, and learning is very slow. The recall is too low, because the probability of a phrase reoccurring literally is too low.

In order to achieve precision, we shall use complete NP's and VP's as terms. However, in order to retain recall we introduce a number of normalizations and a matching techniques, as described in the following sections.

\subsection{The DORO project}

A problem faced by a growing number of companies in the Information Age is the routing of human-readable documents which are in electronic form. Such documents can either be recieved in the form of Electronic messages, or they 
can be the result of OCR-processing of paper mail and documents. How can it be assured that they arrive at the proper destination within the company with the smallest possible time delay?

For properly addressed mail this is just a matter of logistics, but for generically addressed mail and for documents without specific addressing information, this involves at present some form of human intervention: reading the document, interpreting its contents, and forwarding it to the person or department that should deal with it. Manual mail routing is error prone and time consuming. How can it be automated?

The goal of the DOcument ROuting project DORO (Esprit 22716) (see also http: / / www . cs . kun . nl / doro/) is to develop a system performing the automatic routing of human-readable documents in electronic form on the basis of a superficial analysis of the contents of the documents and knowledge of the characteristics of the possible destinees. An overview of the system is shown in Fig. 1. Incoming document images are first separated into forms (which are sent directly to the Work Flow system) and other (typewritten or printed) documents, in particular letters. These documents are OCR converted, classified by the Linguistic Classification system LCS, Workflow Relevant Data (WRD) is extracted and the result passed to the Work Flow system. Documents found to be misclassified are returned through the Work Flow system, and used to adjust the classifiers.

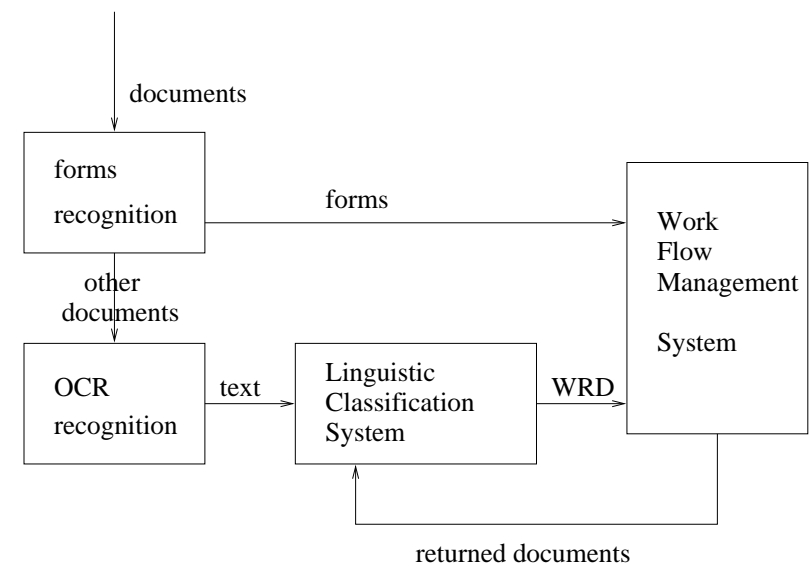

Figure 1: Overview of the DORO system

The main characteristics of the IR approach taken in DORO are:

- rather than with (short) queries, the system deals with profiles of documents and document classes

- profiles consist of linguistically extracted phrases rather than keywords, to enhance precision

- both noun phrases and verb phrases are extracted

- syntactic normalization is introduced to enhance recall

- frames are produced from the phrases by syntax-directed transduction techniques

- fuzzy matching on the basis of semantical word relations is used to enhance Recall

- automatic learning techniques are used for document classification.

The DORO document routing system is intended to bridge the gap between the OCR scanning of documents and the Work Flow system of the company, enabling the fully automatic input and routing of free-format texts. More in general, it can be used for all kinds of filtering, routing and "narrowcasting".

The DORO Consortium is composed of: industrial partners from the OCR and Document Flow areas, providing up to date technology in those areas and serving as integrators; academic partners providing the relevant technologies and 
resources in Natural Language Processing and Information Retrieval; and Users who face a growing document routing problem in their business.

Versions of the system are being developed for three Community languages (Dutch, Spanish and Greek) and three applications (a major Dutch insurance company, the largest Spanish department store and the Athens stock exchange).

\subsection{Linguistic resources}

In the course of the DORO project, grammars for three natural languages have been developed:

- A grammar of Dutch (a further development of the AMAZON grammar [3]), in collaboration between the Computer Science Department of the University of Nijmegen and the Department of Language and Speech.

- A grammar of Spanish, developed by the group of Guillermo Rojo and Paula Santalla at the faculty of Filology, University of Santiago de Compostela

- A grammar of Modern Greek, developed by the group of Dora Noussia at the Computer Technology Institute (CTI) in Patras.

These grammars have been written in the AGFL formalism ("Affix Grammars over a Finite Lattice", see [8]), from which the AGFL system (see http://www.cs.kun.nl/agfl/) generates parsers. Furthermore, heavy use is made of the transduction mechanism of the AGFL system which allows the description of compositional transductions in the grammar: a translation is (recursively) defined for each rule, as a sequence of symbols composed out of the transductions of its elements and inserted symbols (e.g. SGML markers).

For each language, an extensive general lexicon has been compiled, based for as far as possible on existing lexical material. Furthermore, for each language and application in the DORO project a domain lexicon is being developed, providing morphological, syntactical and semantical information about typical domain words and collocations.

\section{Syntactic normalization}

Natural languages allow the expression of one same thought or concept in very many different ways. In fact, we avoid literal repetitions of phrases, making use of linguistic variation for stylistic reasons.

By linguistic normalisation we mean the process of undoing linguistic variation, mapping different but equivalent formulations onto one same representative formulation. Linguistic variation comprizes

- morphological variation, e.g. inflection, traditionally normalized by stemming

- syntactic variation, e.g. the alternative use of phrases like air pollution, polluted air and pollution of the air

- semantic variation, by choosing related words (noxious gases) or periphrasis (dust-laden clouds)

- anaphora (e.g. the pollution in a context relating to air), which is still outside our linguistic scope.

The goal of syntactic normalization is, for as far as this is possible without "deep" semantic analysis, to map syntactically different but semantically equivalent phrases onto one same frame.

The idea of syntactic normalization can already be found in [6]. In this section we briefly describe the syntactic normalization performed by the DORO system.

\subsection{The noun phrase}

Semantically, the noun phrase is a reference to or a description of a thing or a fact - a natural subject for search in a retrieval-based information system. Noun phrases are therefore an accepted term representation in a number of IR systems, and the idea of normalization can be found in e.g. [16] where it is called the conflation of noun phrases. 


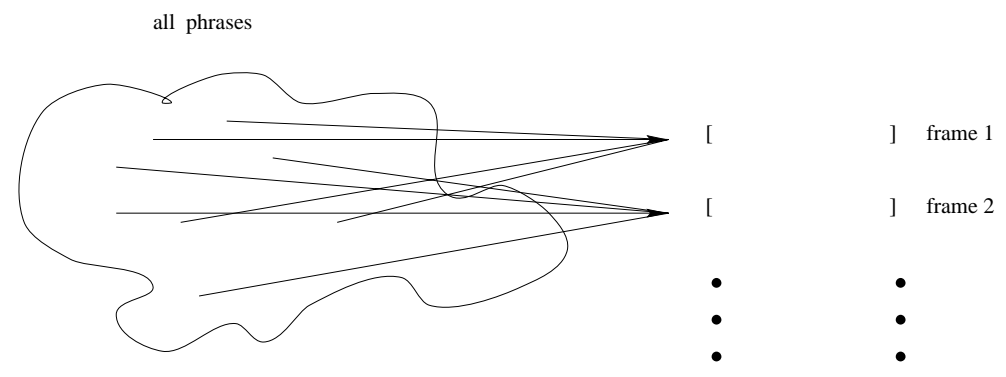

Figure 2: Syntactic normalisation

\subsubsection{The structure of the Noun Phrase}

A noun phrase is roughly that which can occur as the subject or object of a sentence, for instance:

- software engineering conference

- the air pollution in industrial activity zones

- a threshold, which should not be exceeded

Syntactically, a noun phrase may consist of at least the following elements:

1. it starts with zero or more determiners: an article (a or the), a quantifier (e.g. all or many), a number, a demonstrative or possessive pronoun (this, my), or certain combinations of these

2. it contains as its head a noun, a name or a personal pronoun; in the examples the heads are the nouns conference, pollution and threshold

3. the head may be preceded by some pre-modifiers: an adjective like industrial, which may in its turn be modified by an adverb, or a noun, like air in air pollution or even another NP, like software engineering

4. the head may be followed by some post-modifiers: a post-adjective like general in director general (very rare in English), a preposition phrase $(P P)$ like in industrial activity zones, or a relative clause like which should not be exceeded.

Notice that a noun phrase may recursively contain another, or even a (nearly) complete sentence, like in the last example. Therefore, an NP may be arbitrarily complex. Furthermore, we have ignored the coordination between NP's or between their components by means of conjunctors like and and or.

\subsubsection{Normalizing the Noun Phrase}

Syntactic normalization of noun phrases is obviously dependent on the language to which it is applied. The following normalizing transformations come to mind:

- Elimination of determiners (articles, quantifiers and numbers) and adverbs

- Elimination of case distinctions (important for highly inflected languages like Greek)

- English (in particular its american variant) has an unnerving tendency to use nouns as premodifiers to avoid a postmodifier with a preposition (in particular of). For example, the phrases

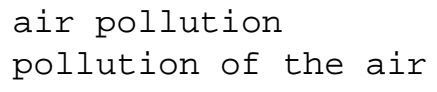


have the same meaning and therefore one should be mapped on the other.

- Unnesting of embedded NP's, examples in section 3.4.

- Other transformations should allow a sensible treatment of coordinated elements. We shall not go into further detail.

All in all, the Noun Phrase presents only few opportunities for normalizing transformations.

\subsection{The Verb Phrase}

The verb phrase is the largest constituent of the (simple) sentence. It comprizes a verb form together with its complements. Semantically, a verb phrase can be seen as the description of a fact or event. It can describe something dynamic, in contrast to the noun phrase which describes something static. Verb phrases should be terms for retrieval in their own right.

Furthermore, it is hard to find the boundaries of NP's without determining the VP structure:

- two NP's may follow one another

In software engineering conferences abound.

- in English many verb forms may be (mis)taken for a noun, or vice versa

Time flies like an arrow.

That is why Noun Phrase Pickers can never be precise enough.

The robust and complete syntax analysis performed in the DORO system provides us with quite precise NP's and VP's (apart from the well-known problems with ambiguity and attachment, which require further progress in NLP).

\subsubsection{The structure of the Verb Phrase}

The verb phrase $(V P)$ is particularly rich in linguistic variation, but the underlying structure of the VP is quite simple and elegant: it consists of a verbal clause which is a (possibly inflected) form of some verb (the main verb) composed with other (auxiliary) verb forms and/or clitics (possibly even distributed over the sentence) accompanied by one or more complements, each fulfilling a certain role: the subject and (possibly) object, indirect object, preposition complement, etc. Complements can be realized by an NP (in the wide sense, including personal names, personal pronouns etc.) or by a PP. Some examples:

$\begin{array}{rrl}\text { I } & \text { subject } \\ \text { would have liked to hit } & \text { verbal clause } \\ \text { the man } & \text { object } \\ \text { prep. complement } & \text { (instrumental) } \\ \text { with a hammer. } & \\ \text { my husband } & \text { subject } \\ \text { me. } & \text { object } \\ \text { I } & \text { subject } \\ \text { owe } & \text { verbal clause } \\ \text { you } & \text { indirect object } \\ \text { does understand } & \text { verbal clause } \\ \text { twenty dollars. } & \text { object }\end{array}$

21st BCS IRSG Colloquium on IR, Glasgow, 1999 


\subsubsection{Normalizing the verb phrase}

For the verb phrase, many more normalizing transformations can be found which preserve meaning, or rather: which have a normalising effect and do not lose information which is obviously relevant for retrieval purposes.

1. elimination of irrelevant elements, like adverbs.

2. depassivation

Abstracting from the distinction between active and passive forms.

3. elimination of time and modality

The verbal clause of the verb phrase can be reduced to the infinitive of the main verb (with clitics). It may make sense to retain an indication of certain modalities (present or future, wish or fact).

4. word-order normalization

Eliminating topicalization and free word order by bringing the complements of each VP in some standard order, like

$$
\text { mainverb subject }[\text { object }][\text { indirect object }] \ldots
$$

5. unnesting of the complements, reducing each NP complement to its head plus a separate NP frame.

The result of all these transformations is a VP frame, an abstraction from the verb phrase suitable for retrieval purposes, with possibly a number of accompanying NP frames.

\section{Phrases, frames and terms}

By phrases we mean certain large constituents of a sentence, represented e.g. by a parse tree. A frame is an abstraction from a phrase, suitable for IR purposes. Frames may be nested. We shall use only frames without nesting as terms for classification, so nested frames will have to be unnested. Both a document class and an individual document are represented by a profile, a set of weighted terms.

The process of extracting terms from a document text consists of a number of steps, as shown very schematically in figure 3.

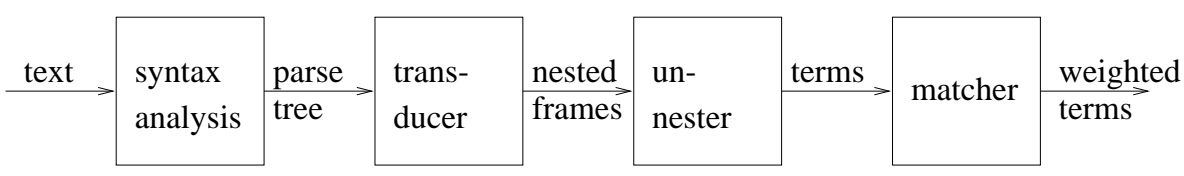

Figure 3: From documents to terms

The syntax analysis transduces the text to a sequence of parse trees for the individual sentences, containing the phrases as subtrees. It uses a parser obtained from an AGFL grammar and a lexicon. It produces parses in an SGML format. The transducer, which is also described by an AGFL grammar, performs the syntactic normalization and transduces each phrase into a (possibly nested) frame. A frame is an abstraction from a phrase, as described in this section. The unnester (described in section 3.4) unfolds the nested frames to obtain the terms describing the document. Finally, the matcher (4) performs a limited form of semantical normalization, using general and domain lexica, and computes the similarity between terms occurring in the document and those occurring in the class profiles. 


\subsection{Frames}

How should we represent phrases in order to use them as terms? A large number of possibilities offer themselves.

On the one hand a phrase can be represented as a parse tree. This provides certainly too much detail, much of which is redundant or irrelevant for retrieval. At the other extreme, it can be represented as a sequence or even set of words. This gives too little detail, since essential information about the meaning is not represented. For example, it is no longer visible what noun is the head of the noun phrase. In fact we can take all kind of intermediate forms.

The actual production of frames is performed by a separate transducer (described by another grammar), which takes as input the trees produced by the parser (coded in SGML). This separates the issues of language description and frame production.

The question which parts of a phrase to express in the form of frames and how is left entirely to the grammar writer. The system merely provides a general notational and implementational framework. Experimentation will have to decide what is the best choice. In this section we will outline the general approach.

\subsection{Headedness}

According to the principle of headedness, any phrase has a single word as head (usually a noun, a name or a personal noun in the case of an NP, the main verb in case of a VP). The notion of head is common to most linguistic theories and by itself relatively language independent.

Phrases with the same head are related. The head gives the central concept of the phrase, and the other elements (modifiers in the NP, complements in the VP) serve to make it more precise. Conversely, the head may be used as an abstraction of the phrase (losing precision but gaining recall).

\subsection{Binary frames}

Rather than introducing general tree structures as terms, we shall use frames, defined as pairs [head, modifier], where the head is a nonempty string (a lemmatized word) and the modifier is an (arbitrarily long) string of lemmatized words, which is empty in the case of a bare head. This last case may also be denoted by [head].

The head also serves as an index into a list of frames with frequencies etc., organised into groups with the same head like

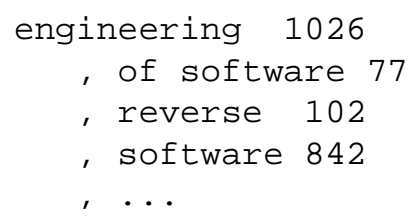

In order to keep the examples readable, we do not show the lemmatization and tagging information attached to the words. Note that the frequency of the head includes the frequencies of its modified occurrences.

The result of the transduction of a parse tree is a sequence of frames (including single symbols and nested frames), according to the following syntax (somewhat simplified):

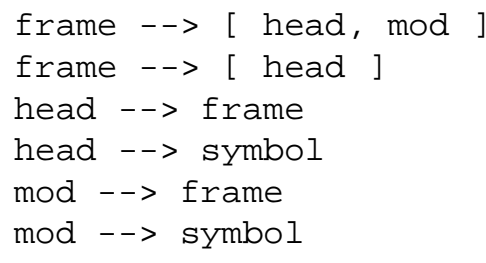

As an example, the sentence

he visited a conference on software engineering

may be transduced to the nested frame

[visit, [conference, [engineering, software]] ] 


\subsection{Unnesting}

In order to raise recall, we follow the strategy of unnesting all nested frames: a composed frame like [c, [b, a]] will be decomposed into two frames $[\mathrm{b}, \mathrm{a}]$ and $[\mathrm{c}, \mathrm{b}]$, using the head $\mathrm{b}$ as an abstraction for $[\mathrm{b}, \mathrm{a}]$ in the second one. More in general, $\alpha[h, \bmod s] \beta$ is replaced by $\alpha h \beta$ while introducing a separate frame $[h$, mods $]$.

Unnesting is applied recursively to the head and modifiers of a frame. In this way, a frame containing frames is rewritten into a collection of frames without nesting, which are then used as terms. A single symbol $\mathrm{x}$ is rewritten into a term $[\mathrm{x}]$. As an example, the nested frame

[visit, [conference, [engineering, software]] ]

is unnested to

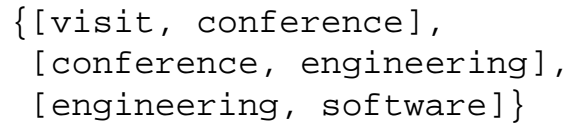

Another example: the (in)famous phrase the Hillary Clinton Health Care Bill proposal

resulting in a nested frame

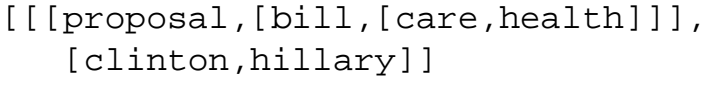

will be unnested to the collection of frames

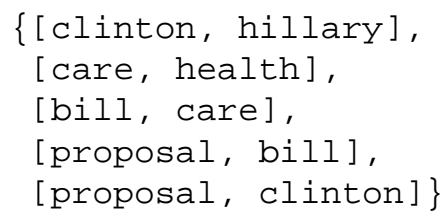

which does not include [clinton, bill].

The result is that from one phrase a (multi)set of frames without nesting is produced as terms, rather than a single nested one. This strategy raises the recall and looses some precision. Of course the unnesting makes it all the more important that the parser should be able to deduce the appropriate dependency structure in complicated phrases.

\section{Matching}

The matching process comprizes the following tasks:

1. syntactic matching (partial matching of composed terms), and

2. semantical matching (exploiting synonymy and hypernymy relations).

In our matching strategy we exploit certain properties of the classification algorithms.

\subsection{Relevant properties of the classification algorithms}

The classification algorithms used in the project [10] have the property that they compute a linear classifier in the sense that the similarity between a document profile and a class profile (each of which is given as a (multi)set of terms), is calculated as the sum of the weights given to those terms that are present. They perform best when faced with a low number of terms with high predictive value.

It is well known that, as the number of documents grows, the number of different keywords occurring in them grows approximately with the square root of the number of documents. The number of phrases however grows even faster than the number of words. This has negative consequences for the classification algorithms, negating the positive effect of having more precise terms: 
- a large number of terms with hard to tract dependencies between them

- a low probability of any particular term reappearing literally in another document.

\subsection{The matching strategy}

The conventional approach to syntactic matching of phrases is to define some similarity function between phrases, based e.g. on subtree overlap. This function should then be used to weigh the phrases in the classification algorithms.

We have studied this approach, but found it hard to define a reasonable measure. Instead, we have introduced unnesting to obtain from a composed phrase only those terms which are considered relevant from a linguistic point of view. This may be more efficient and is certainly more tractable than subtree matching. Moreover, the experience collected with the classification algorithms has made clear that applying syntactic matching to composed terms and all their subterms is not a good strategy: One would have to combine in an ad-hoc fashion fractional contributions from very many terms. It is better to rely on the property of the algorithms, firmly founded in probability theory, of being able to make their own judgement as to the significance of individual terms. And how should one count the frequencies of partially matched terms?

Once a term has occurred an appropriate number of times in documents belonging to specific classes, the judgement of the classification algorithm can be trusted. The problem is how to deal with terms that have not occurred before, or have not yet achieved significance. It is in this "fringe" that similarity to other terms becomes significant.

Our strategy will be to apply semantical matching only to terms that are insignificant according to the classification algorithm, trying to find the most significant related term. This closest term is then used by the classification, but the original term is used by the learning algorithm. Thus, given enough documents the term may become significant later. A more precise formulation of this (literally) marginal strategy will be found in section 4.6.

\subsection{Term significance}

Intuitively, a term is significant if its presence or absence makes a difference in the outcome of the classification process. Due to the linear character of our classification algorithms, significance is a gradual scale: some terms will be more significant than others. What terms are significant, and to what degree, depends to a certain extent on the classification algorithm used, but in all cases a term that has not occurred in a training document will have no significance, and a term that occurred only once or in only one document will have very little. In fact, the proper treatment of insignificant terms presents a problem for most classification algorithms. It is preferable to have a small number of highly significant terms rather than a large number which are of doubtful significance.

In the Machine Learning community, where many classification algorithms originate, a number of techniques have been invented to perform "feature selection". In applying such techniques to text classification [18] it was found that the number of features (in our case terms) could be reduced by a factor of ten without impairing classification accuracy, or even slightly improving it.

Our own experiments with two dynamic stoplist heuristics (Winnow steps and a frequency heuristic) show similar results [10]. Applying three winnow steps to the SBC algorithm raised in our main experiment the overall precision from $80 \%$ to $84 \%$, while reducing the number of terms by a factor eight.

After training a classifier, it may be much reduced in length by eliminating all insignificant terms from it. This allows the classification in the production phase to be more economical in time and space. It is only during training that classifiers over the full set of terms are needed. The process of searching a classifier for semantical matching also is speeded up by using shorter classifiers.

\subsection{Lexico-semantical relations}

For the DORO project we have decided to adopt (Euro)WordNet [17] as the source of general lexico-semantical data for semantical matching, using only synonymy and hyper/hyponymy (other relations are available, but their value is unclear). In WordNet, a word with a certain Part-Of-Speech (POS) category is collected with its synonyms into a synset. A synset can be seen as the representation of a concept. 
Besides a word, a synset may also contain a reference to a hypernymous word, indicated by a word marked with @, and a reference to a hyponym, indicated by a word marked with ; from this word, again its synset can be taken. Thus, the WordNet data structure can be characterized by three operations:

$$
\begin{aligned}
& \text { syn }: \text { word } \times \text { POS } \rightarrow\{\text { synset }\} \\
& \text { hyper }: \text { synset } \rightarrow\{\text { synset }\} \\
& \text { hypo : synset } \rightarrow\{\text { synset }\}
\end{aligned}
$$

where $P O S \in\{N O U N, A D J, V E R B\}$ and synset $=\{$ word $\}$. The domain lexica developed within the project are structured similarly. In the examples the POS will not be shown for simplicity.

\subsection{Domains and senses}

The notion of synonymy in WordNet means substitutional equivalence in a certain context, and synsets are distinguished according to context. Polysemous words are distinguished by word sense. In recent papers on Retrieval applications using WordNet [13, 7], the importance of word sense disambiguation has been stressed. But there are no reliable techniques for automatic word sense disambiguation.

In Text Classification, the queries (being complete documents) are much longer than in classical Retrieval. Furthermore, in our applications the documents are restricted to a limited domain (e.g. letters to an insurance company). Therefore there may be less need for word sense disambiguation. Instead, we will give higher priorities to words and synsets that are related to the application domain, distinguishing three lexico-semantical levels in decreasing priority:

1. the domain lexicon, constructed (in the style of WordNet) for the words in the particular application domain;

2. the semi-domain lexicon, containing words and synsets taken from the WordNet which are relevant for the application domain, and

3. the general lexicon comprizing the remaining words and synsets.

The rationale behind this division is that it allows an exploitation of the domain restriction of the documents to achieve some measure of word sense disambiguation.

\subsection{Semantical matching of terms}

The input to the matcher is a sequence of terms, with the syntax

term --> [ symbol, symbol]

term $-\rightarrow$ [ symbol]

where a symbol is a word together with its POS.

Our matching strategy, applied only to those terms which are not already significant according to the class profiles, is that of selective expansion.

By the expansion of a word with a certain POS we shall mean that word together with its synonyms and its direct hyper- and hyponyms.

$$
\begin{aligned}
& \exp : \text { word } \times P O S \rightarrow\{\text { word }\} \\
& \exp (w, p)=\{w\} \cup \text { flatten }(\operatorname{syn}(w, p)) \cup \\
& \quad \text { flatten }(\text { hyper }(\operatorname{syn}(w, p))) \cup \text { flatten }(\operatorname{hypo}(\operatorname{syn}(w, p)))
\end{aligned}
$$

As an example, the (simplified) synset for tree

\{ tree, olant, conifer \} 
through the hyponym pointer $\sim$ plant points to the synset

\{ plant, flora, organism, etree \}

(note the corresponding hypernym pointer to tree for plant). The expansion of tree is the extended set

$$
\text { \{ tree, plant, flora, organism, conifer \} }
$$

By the expansion of a term we mean the set

$$
\begin{aligned}
& \exp : \text { term } \rightarrow\{\text { term }\} \\
& \exp ([x])= \\
& \{[t] \mid t \in \exp (x)\} \\
& \exp ([x, y])=\exp ([x]) \cup \\
& \{[t, u] \mid t \in \exp (x), u \in \exp (y)\}
\end{aligned}
$$

The semantical matching of an insignificant term consists in its replacement by the most significant term in its expansion.

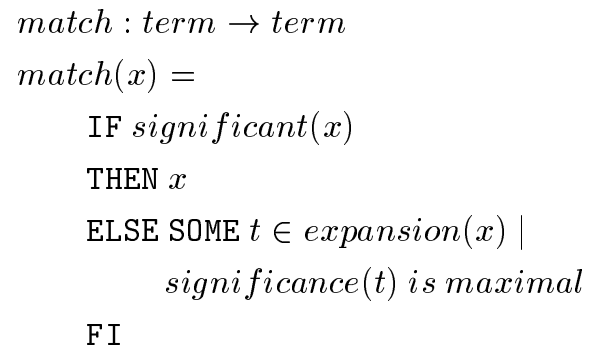

Insignificant terms having no significant expansion are dropped. For efficiency reasons, the set of all terms related to a significant term may be precomputed.

The terms obtained by expansion have to be weighted by some suitable constant $\lambda$ (e.g. $\lambda=.9$ for synonyms and $\lambda=.5$ for direct hyper/hyponyms).

The semantical matching technique described here is related to the traditional technique of "massive expansion" of queries, but with the difference that we do not extend the query with all (syno-, hyper-, hypo-) nyms, but rather replace insignificant terms by their single most significant nym.

\section{Conclusion}

We have described a number of techniques for using phrases as terms in a Text Classification system:

- syntactic normalization through the transduction and unnesting of binary frames, and

- lexico-semantical matching through the selective expansion of terms considered insignificant by the classification algorithm.

For the benefit of other reasearchers we have described the rationale behind our design decisions, leading to a conceptually simple and rather general classification system. We are still building the DORO system and can not yet provide experimental results. Further experimentation will be necessary to achieve an optimal linguistic support for Text Classification. 


\section{References}

[1] A.T. Arampatzis, T. Tsoris, C.H.A. Koster and T.P. van der Weide (1988), Phrase-based Information Retrieval. Information Processing \& Management, 34(6), pp. 693-707.

[2] W.W. Cohen and Y. Singer (1996), Context-sensitive methods for text categorization. Proceedings SIGIR'96, pp. 307-315.

[3] P.-A. Coppen (1995), A new version of the Amazon/Casus system. In: Proceedings of the Department of Language and Speech, P. de Haan and N. Oostdijk (eds.), part 18, pp. 85-90. University of Nijmegen, 1994.

[4] I. Dagan, Y. Karov and D. Roth (1997), Mistake-Driven Learning in text Categorization. In: Proceedings of the Second Conference on Empirical Methods in NLP, pp. 55-63.

[5] D.A. Evans, R.G. Lefferts, G. Grefenstette, S.H. Handerson, W.R. Hersch and A.A. Archbold (1993), CLARIT TREC design, experiments and results. In: TREC-1 proceedings, pp. 251-286.

[6] J.L. Fagan (1988), Experiments in automatic phrase indexing for document retrieval: a comparison of syntactic and non-syntactic methods, PhD Thesis, Cornell University.

[7] J. Gonzalo, F. Verdejo, I. Chugur an J. Cigarrán (1999), Indexing with WordNet synsets can improve text retrieval, Proceedings COLING/ACL 1999.

[8] Cornelis H.A. Koster (1992), Affix Grammars for Natural Languages. In H. Alblas and B. Melichar, editors, Attribute Grammars, Applications and Systems, volume 545 of Springer LNCS, pp. 469-484.

[9] M.F. Porter (1980), An algorithm for suffix stripping. Program, 14, pp. 130-137.

[10] H. Ragas and C.H.A. Koster (1998), Four classification algorithms compared on a Dutch corpus. Proceedings SIGIR'98, pp. 369-370.

[11] C.J. van Rijsbergen (1979), Information Retrieval. 1979, London, Butterworths.

[12] J.J. Rocchio (1971). Relevance feedback information retrieval. In: Salton, G. (ed.) The smart retrieval systemexperiments in automatic document processing p. 313-323. Prentice-Hall, Englewood Cliffs, NJ.

[13] A.F. Smeaton and I. Quigley (1996), Experiments on using semantic distances between words in image caption retrieval, Proceedings SIGIR'96.

[14] A.F. Smeaton (1997), Using NLP and NLP resources for Information Retrieval Tasks. In: T. Strzalkowski (Ed.), Natural Language Information Retrieval, Kluwer Academic Publishers.

[15] K. Sparck Jones (1998), Information retrieval: how far will really simple methods take you? in: Proceedings TWTL 14, Twente University, the Netherlands, pp. 71-78.

[16] T. Strzalkowski, F. Lin and J.P. Carballo (1997), Natural Language Information Retrieval: TREC-6 Report. To appear.

[17] P. Vossen (Ed.) (1998), EuroWordNet A Multilingual Database with Lexical Semantic Networks. Kluwer Academic publishers.

[18] Yiming Yang and Jan Pederson (1997), Feature selection in statistical learning of text categorization. In: ICML 97, pp. 412-420. 